\title{
Theoretical investigations on the geometric and electronic structures of polyacetylene molecule under the influence of external electric field
}

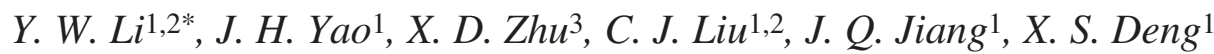

${ }^{1}$ Key Laboratory of New Processing Technology for Nonferrous Metals and Materials, Ministry of Education, Guilin University of Technology, Guilin 541004, PR. China

2Department of Material and Chemical Engineering, Guilin University of Technology, Guilin 541004, P.R. China

${ }^{3}$ Department of Applied Chemistry, Harbin Institute of Technology, Harbin, 150001, PR China

Received 12 July 2009; accepted in revised form 13 August 2009

\begin{abstract}
The geometric and electronic structures of all-trans polyacetylene (PA) molecule in neutral, cationic, and anionic states have been studied theoretically by density functional theory method at the B3LYP/6-31+G* level. The results show that both the geometric and electronic structures of the PA molecule are sensitive to the external electric field (EF). For neutral PA molecule, with the increase of EF, the carbon-carbon single bonds are shortened while the carbon-carbon double bonds are elongated. The energy gap between the highest occupied molecular orbital and the lowest unoccupied molecular orbital (LUMO-HOMO gap) decreases with the EF increasing. For cationic PA molecule, the carbon-carbon single bonds and carbon-carbon double bonds on the high potential side are elongated and shortened, respectively. While, the carboncarbon single bonds and carbon-carbon double bonds on the low potential side are shortened and elongated, respectively. Contrary to the neutral PA case, the LUMO-HOMO gap increases with the EF increasing. Contrast to the case of cationic PA, the evolution of carbon-carbon bond lengths for the anionic PA molecule under the external EF reverses. The LUMOHOMO gap of the anionic PA molecule decreases with the increase of external EF. In addition, the spatial distributions of the HOMO and LUMO under the influence of external EF are also discussed for the PA molecule in neutral, cationic, and anionic states.
\end{abstract}

Keywords: modeling and simulation, density functional theory, polyacetylene, electronic structure, geometric structure

\section{Introduction}

$\pi$-conjugated polymers are the subject of recent fundamental and industrial research due to their novel properties and a variety of applications in the electronic devices, such as light-emitting diodes (LED) [1, 2], field-effect transistors (FET) [3, 4], and molecular electronic devices $[5,6]$. The characteristic feature of all these substances is the conjugated double bond. Among these conjugated polymers, all-trans polyacetylene (PA) is the most prominent representative of this kind of material, and many other conjugated polymers can, in principle, derived by the structure modification. Therefore, it has been studied intensively both experimentally [7-9] and theoretically [10-12].

Previous works [13-15] demonstrated that the properties of these polymers are critically dependent on the geometric and electronic structures of the molecules. Therefore, knowledge about these characters of the conjugated molecules is highly desired not only for understanding the packing model of molecules in solid, but also for designing or ration- 
alizing electronic devices. Although much information of the properties for this kind of materials has been obtained with the assistance of nuclear magnetic resonance (NMR) [16], X-ray analysis [17], and scanning tunneling microscopic (STM) investigation [18], the details of the molecular geometric and electronic structures as well as the structure/ properties relationships are not very clear. Electric conductance is a bulk property. However, it does indeed take place over the single molecules [19]. Moreover, conjugated molecules are promising materials for electronic devices [1-6, 20], in which the molecules undergo a large external electric field (EF). Thus, in order to study, and eventually to be able to predict, electrical conduction of polymers made from organic monomers, it is important to understand the nature of the molecular response to an external EF both for neutral and charged species. However, there is no systematic report on these issues yet. The purpose of this work is to perform theoretical investigations on the evolution of the geometric and electronic structures of PA molecule in neutral and charged states by considering the influence of external EF.

\section{Methods}

Previous theoretical investigations of conjugated molecules have demonstrated the significance of correlation effects [21, 22]. In order to perform an accurate calculation on the geometric and electronic properties of a conjugated molecule, the computation of electron correlation effects by many-body perturbation theory should be carried out after the Hartree-Fock (HF) calculation. Alternatively, we can do density functional theory (DFT) calculation, in which the electron correlation is taken account. In the present work, all the calculations are performed at the B3LYP level with $6-31+\mathrm{G}^{*}$ basis set, which is widely used for calculations for conjugated molecules [22, 23], by using the GAUSSIAN 03 program [24].

Theoretical modeling has been achieved as follows. Prior to the introduction of EF, linear chain of alltrans PA (see Figure 1) was fully optimized at the B3LYP/6-31+G* level for both the neutral and charged states. All the geometric parameters were, then, optimized at the same level of theory in the application of uniform external EF. A uniform EF ranging from zero to $2.57 \cdot 10^{9} \mathrm{~V} \cdot \mathrm{m}^{-1}$ and aligned

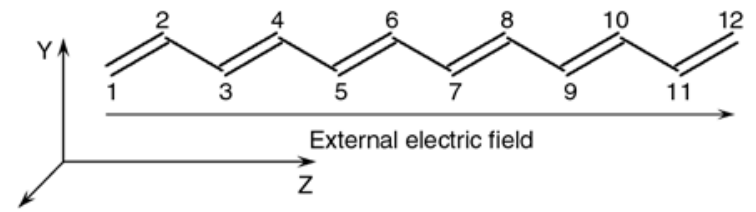

Figure 1. Scheme of the polyacetylene molecule studied in the present work. The external EF is aligned along the two terminal carbon-carbon interatomic vector $\mathrm{C} 1-\mathrm{C} 12$.

along the two terminal carbon-carbon inter-atomic vector was applied to the model molecules (see Figure 1), which may reasonably represent the working condition of organic electronic devices $[19,25]$.

\section{Results and discussion}

\subsection{EF effect on the geometric structure}

The equilibrium geometries of the neutral and charged all-trans PA without EF (zero EF) show a coplanar conformation with $C_{2 \mathrm{~h}}$ symmetry as expected. The carbon-carbon bond lengths along the backbone is one of the most crucial parameters in determining molecular properties, such as static electronic polarizability, second hyperpolarizability [26], nonlinear optical property [27], and the energy gap between the highest occupied molecular orbital (HOMO) and the lowest unoccupied molecular orbital (LUMO) (abbreviated as LUMOHOMO gap) in the conjugated molecules [28]. Figure 2 presents the bond lengths of the model PA in neutral state, cationic state, and anionic state. It

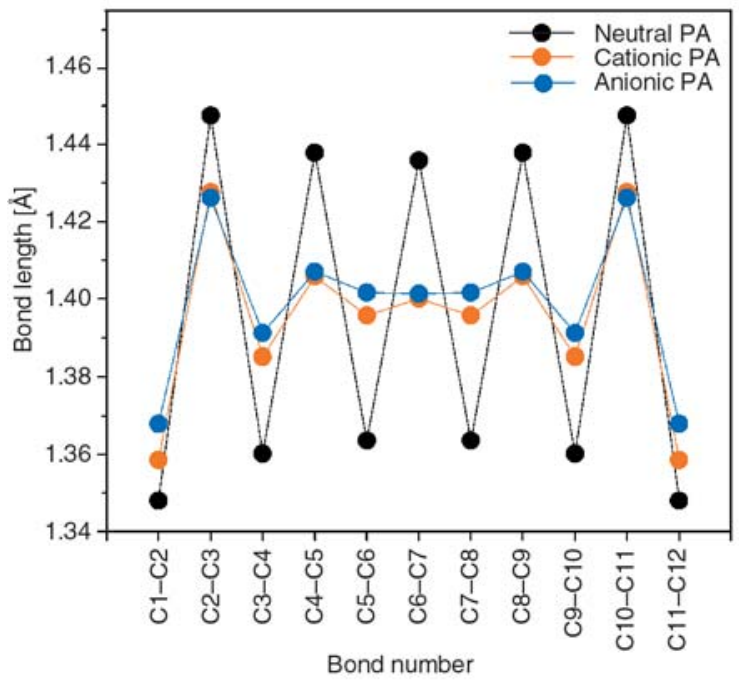

Figure 2. The bond lengths of PA molecule in neutral state, cationic state, and anionic state as a function of bond number (see chemical structures in Figure 1 for bond codes) 
can be seen that the neutral PA shows biggest bond length alternation (BLA, the average of the difference in the length between the adjacent carbon-carbon bonds in the polyacetylene). When the PA gets charged, the BLA is decreased dramatically. In addition, the BLA of the anionic PA is somewhat smaller than that of the cationic PA. The carboncarbon bond length is predominated not only by the nature of the molecule, but also the surroundings. Especially, when a considerable EF is applied, the carbon-carbon bond length is expected to vary [29]. In order to study, and eventually to be able to predict the properties of the conjugated molecule, it is important to understand how the carbon-carbon bond length responds to the external EF.

After the application of EF, the molecular symmetry is destroyed, although the coplanar conformation remains. Figure 3 gives the evolution of the bond length with respect to that of zero EF for the model molecule (see Figure 1) in neutral state. When EF increases, the carbon-carbon single bonds become shorter and the double bonds become longer, resulting in a decreased BLA. Similar result has also been observed for other conjugated molecules, such as polythiophene and oligo(phenylene ethylene), as reported by us [30, 31]. However, the EF dependence of the bond length is not identical for all the bonds. The bonds on the high potential side show more deviation than those on the low potential side of the model molecule. In addition, the maximum deviation occurs in the central part

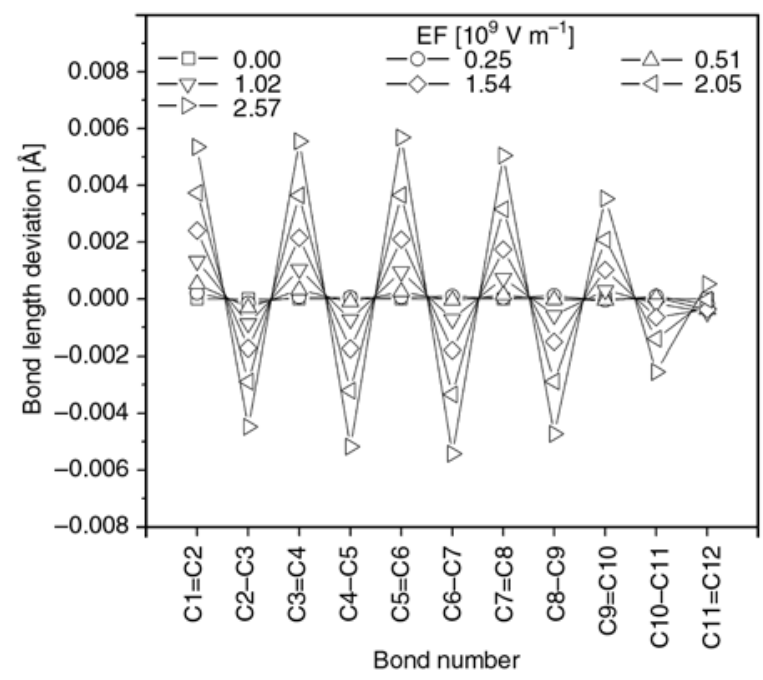

Figure 3. Geometric deviation of the bond lengths of neutral PA molecule under various external EF (see chemical structures in Figure 1 for bond codes). The bond lengths under zero EF are referred to as zero.

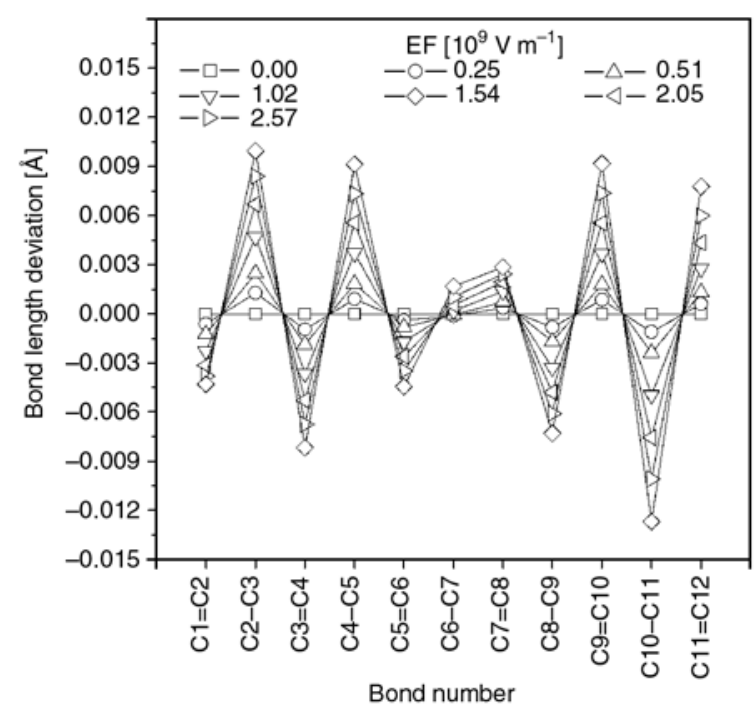

Figure 4. Geometric deviation of the bond lengths of cationic PA molecule under various external EF (see chemical structures in Figure 1 for bond codes). The bond lengths under zero $\mathrm{EF}$ are referred to as zero.

for both the single and double bonds due to the chain end effect [29] and the better conjugation as compared to those toward the end of the molecule. When the molecules are under nonequilibrium conditions, the electronic state would be different and accordingly it is essential to investigate the charged state. Figure 4 presents the evolution of bond length with respect to that of zero EF for the model molecule in cationic state. When EF is introduced, all the bond lengths change. However, the bond length evolution is very different from that observed for the neutral PA. With the EF increasing, the single bonds and double bonds on the high potential side get longer and shorter, respectively; while the single bonds and double bonds on the low potential side get shorter and longer, respectively. Therefore, the application of external EF weakens the conjugation of the high potential side of the molecule and strengthens the conjugation of the low potential side of the molecule. Contrary to the case of neutral PA, the bonds in the central part of cationic PA exhibit minimum bond length deviation. Figure 5 shows the evolution of bond length with respect to that of zero $\mathrm{EF}$ for the model molecule in anionic state. In contrast to the case of cationic state, for the anionic PA, with the EF increasing, the single bonds and double bonds on the high potential side get shorter and longer, respectively; while the single bonds and double bonds on the low potential side get longer and 


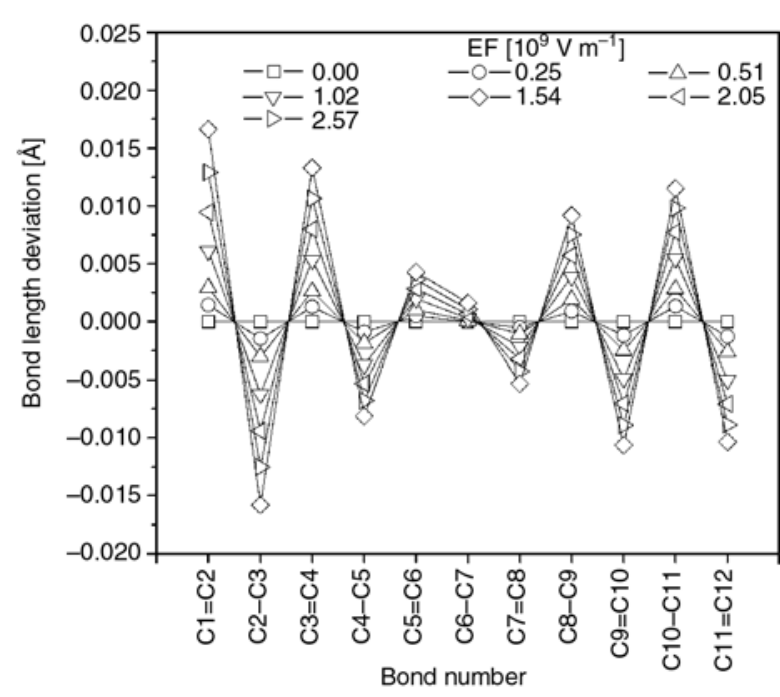

Figure 5. Geometric deviation of the bond lengths of anionic PA molecule under various external EF (see chemical structures in Figure 1 for bond codes). The bond lengths under zero $\mathrm{EF}$ are referred to as zero.

shorter, respectively. Thus, the application of external EF strengths the conjugation of the high potential side of the molecule and weakens the conjugation of the low potential side of the molecule. Similar to the case of cationic PA, the bonds in the central part of the anionic PA exhibits minimum bond length deviation.

\subsection{EF effect on the electronic structure}

The energy levels of the molecular orbitals, especially the HOMO and LUMO, are excellent indicators of many molecular properties, such as chemical activity [32] and electrical performance [33]. Figure 6a gives the HOMO and LUMO levels for the model PA in neutral state calculated by B3LYP/6-31+G* method. It can be seen that with the increase of $\mathrm{EF}$, both the energy levels of HOMO and LUMO decrease. When the EF increases from zero to $2.57 \cdot 10^{9} \mathrm{~V} \cdot \mathrm{m}^{-1}$, the HOMO and LUMO are decreased by 1.66 and $1.90 \mathrm{eV}$, respectively. Figure $6 \mathrm{~b}$ illustrates the LUMOHOMO gap of the model PA in neutral state. Under zero $\mathrm{EF}$, the LUMO-HOMO gap is $2.93 \mathrm{eV}$. With the EF increasing, the LUMO-HOMO gap decreases. By detailed analysis, it is interesting to find that the LUMO-HOMO gap decreases linearly with the square of EF (see the inset in Figure 6b). Figure 7a shows the HOMO and LUMO levels for the model PA in cationic state calculated by
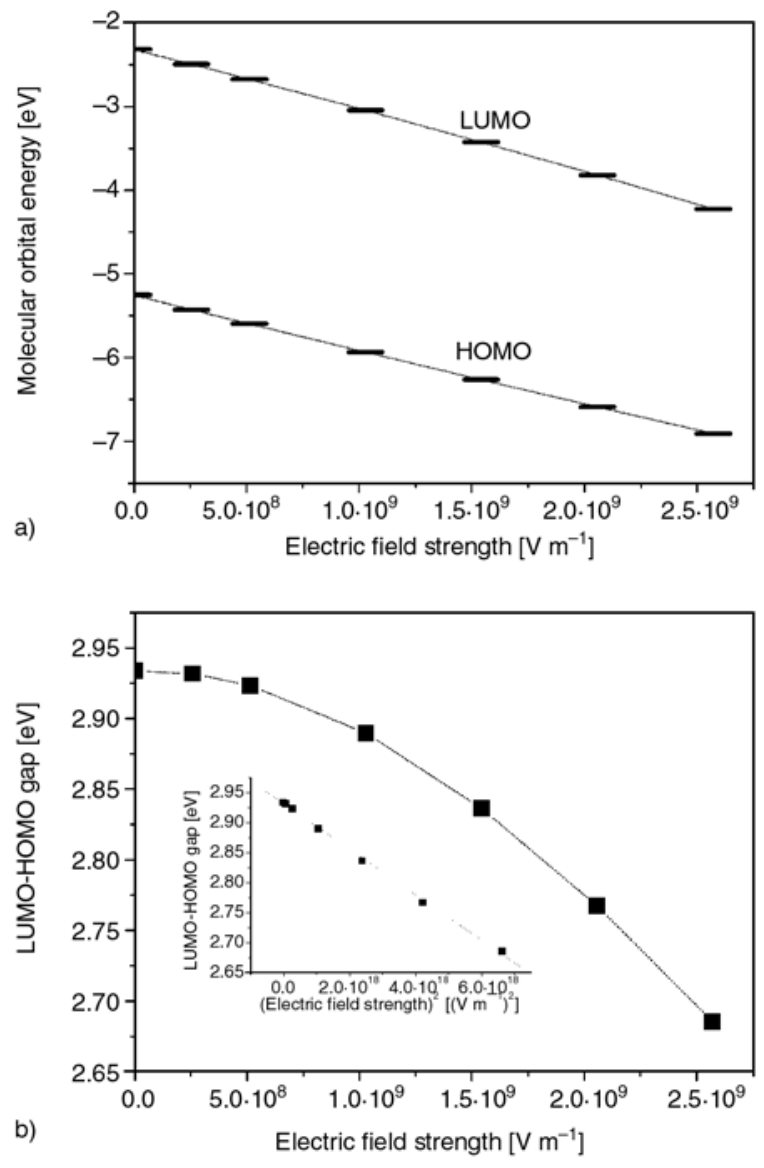

Figure 6. (a) LUMO and HOMO energies for the neutral PA molecule under various external EF;

(b) LUMO-HOMO gap for the neutral PA molecule under various external EF. The inset shows the linear relationship of the LUMO-HOMO gap and the square of EF

B3LYP/6-31+G* method. Similar to that of neutral case, with the EF increasing, both the HOMO and LUMO decrease. When the EF increases from zero to $2.57 \cdot 10^{9} \mathrm{~V} \cdot \mathrm{m}^{-1}$, the decrements of HOMO and LUMO are 1.74 and $1.67 \mathrm{eV}$, respectively. Figure $7 \mathrm{~b}$ gives LUMO-HOMO gap of the model PA in cationic state. Under zero EF, the LUMOHOMO gap is $2.58 \mathrm{eV}$, which is $0.35 \mathrm{eV}$ lower than that of the neutral PA. Contrary to the case of neutral PA, with the EF increasing, the LUMOHOMO gap of the cationic PA increases. A linear relationship between the LUMO-HOMO gap and the square of EF is also observed for the cationic PA (see the inset in Figure 7b). Figure 8a presents HOMO and LUMO levels for the model PA in anionic state calculated by B3LYP/6-31+G* method. With the EF increasing, both the HOMO and LUMO decreases. When the EF increases from zero to $2.57 \cdot 10^{9} \mathrm{~V} \cdot \mathrm{m}^{-1}$, the decrements of HOMO 

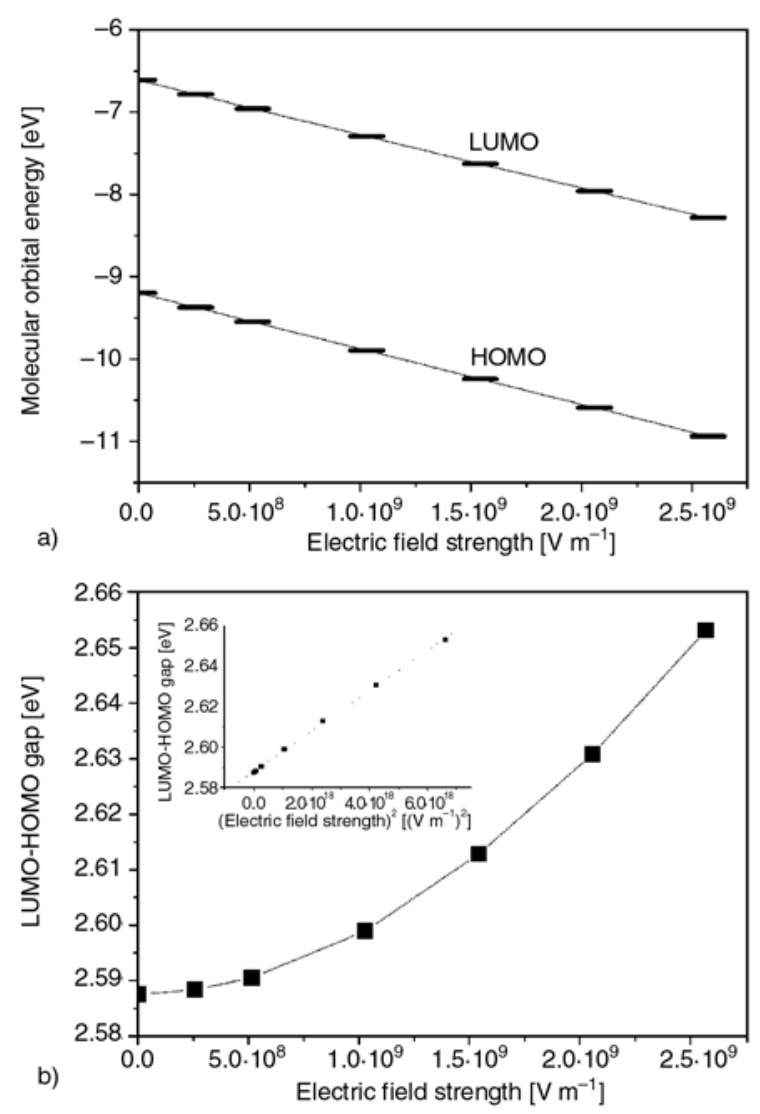

Figure 7. (a) LUMO and HOMO energies for the cationic PA molecule under various external EF;

(b) LUMO-HOMO gap for the cationic PA molecule under various external $\mathrm{EF}$. The inset shows the linear relationship of the LUMOHOMO gap and the square of EF.

and LUMO are 1.91 and $1.94 \mathrm{eV}$, respectively. Figure $8 \mathrm{~b}$ gives LUMO-HOMO gap of the model PA in anionic state. Under zero EF, the LUMO-HOMO gap is $2.42 \mathrm{eV}$, which is 0.16 and $0.51 \mathrm{eV}$ lower than that of the neutral PA and cationic PA, respectively. When EF is less than $0.51 \cdot 10^{9} \mathrm{~V} \cdot \mathrm{m}^{-1}$, the LUMO-HOMO gap seems not sensitive to the charges of EF. However, when the EF is higher than $0.51 \cdot 10^{9} \mathrm{~V} \cdot \mathrm{m}^{-1}$, the LUMO-HOMO gap decreases dramatically. When EF increases from $1.02 \cdot 10^{9}$ to $2.57 \cdot 10^{9} \mathrm{~V} \cdot \mathrm{m}^{-1}$, the LUMO-HOMO gap almost decreases linearly with the increase of EF. Compared to the cases of the neutral PA and cationic PA, the distortion of the linear relationship between the LUMO-HOMO gap and the square of $\mathrm{EF}$ for the anionic PA is possibly caused by the enhanced electron interaction from the additional electron.

Other than the energy levels of the molecular orbitals, one way to understand the molecular prop-
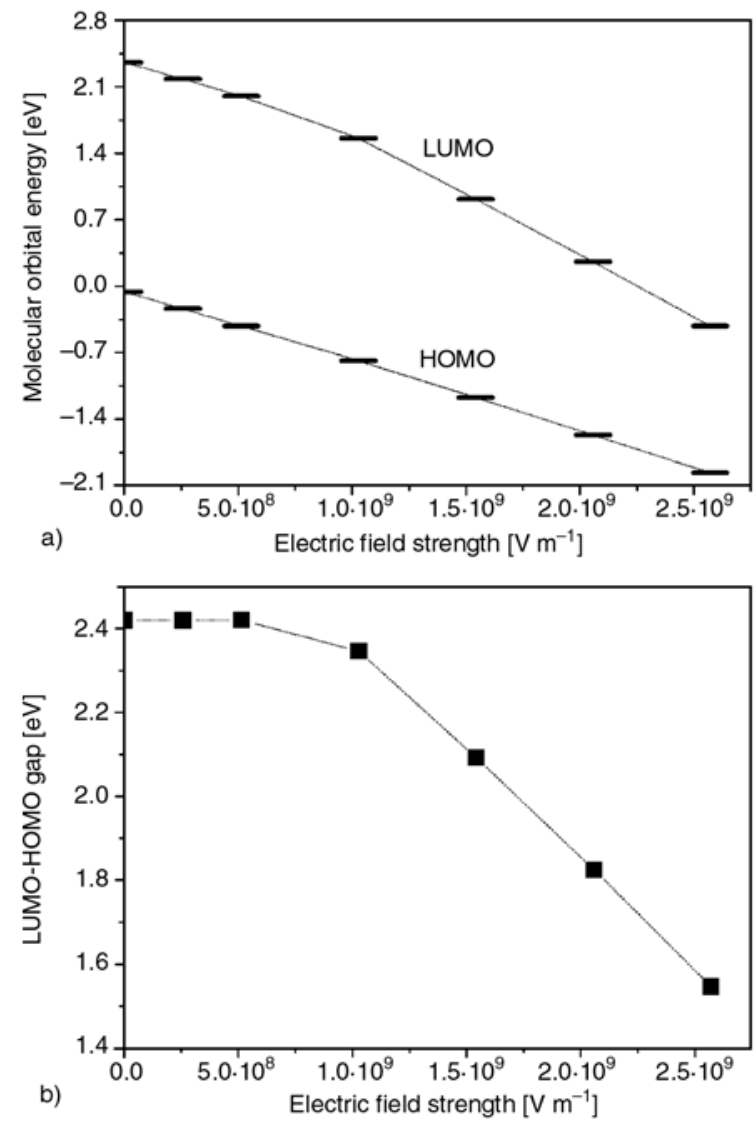

Figure 8. (a) LUMO and HOMO energies for the anionic PA molecule under various external EF; (b) LUMO-HOMO gap for the anionic PA molecule under various external $\mathrm{EF}$

erties is to analyze the spatial distribution of frontier orbitals [34]. Table 1 compares the spatial distribution of the HOMO and LUMO for the model PA in neutral, cationic, and anionic states under various $\mathrm{EF}$. At zero $\mathrm{EF}$, both the HOMO and LUMO for the PA in neutral and charged states are fully delocalized on the whole molecular backbone. For the neutral PA, the introduction of EF tends to move the HOMO to the low potential side and the LUMO to the high potential side. Similar result has also been observed for other conjugated molecules, such as polythiophene and oligo(phenylene ethylene), as reported by us $[30,31]$. While for the cationic PA, both the HOMO and LUMO are almost unchanged with the application of EF. For the anionic PA, the HOMO tends to shift to high potential side with the increasing of EF; The LUMO almost remains when the EF is less than $0.51 \cdot 10^{9} \mathrm{~V} \cdot \mathrm{m}^{-1}$. However, further increasing EF leads the LUMO practically localize on the high potential end of the molecule. 
Table 1. Spatial distribution of HOMO and LUMO of the PA in neutral state, cationic state, and anionic state under various EF

\begin{tabular}{|c|c|c|c|c|c|c|}
\hline \multirow{2}{*}{$\begin{array}{l}\text { EF 10 } 10^{9} \\
{\left[\mathrm{~V} \cdot \mathbf{m}^{-1}\right]}\end{array}$} & \multicolumn{2}{|c|}{ Neutral PA } & \multicolumn{2}{|c|}{ Cationic PA } & \multicolumn{2}{|c|}{ Anionic PA } \\
\hline & HOMO & LUMO & HOMO & LUMO & HOMO & LUMO \\
\hline 0.00 & 'ं0000 & o. oloo & ச́000, & óo yoa & @ & 0,00000 \\
\hline 0.51 & 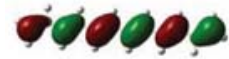 & 000000 & क் & oboua & OQ & 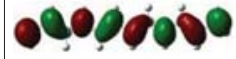 \\
\hline 1.54 & 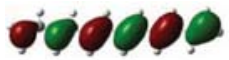 & ooloooa & 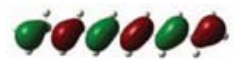 & oo oooa & a & \\
\hline 2.57 & 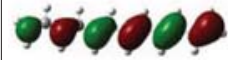 & oouage & テंல்) & oologoo & eace & \\
\hline
\end{tabular}

\section{Conclusions}

B3LYP/6-31+G* calculations are performed to study the geometric and electronic characters of alltrans polyacetylene (PA) molecule in neutral, cationic, and anionic states under the influence of external electric field (EF). For neutral PA molecule, the carbon-carbon single bonds are shortened while the carbon-carbon double bonds are elongated with the EF increasing. The energy gap between the HOMO and LUMO is decreased with the interlocution of the external EF. For cationic PA molecule, the carbon-carbon single bonds and carbon-carbon double bonds on the low potential side are elongated and shortened, respectively. While, the evolution of carbon-carbon bond length on the high potential side reverses. Contrary to the neutral PA case, with the increase of $\mathrm{EF}$, the LUMO-HOMO gap increases. When compared to the case of cationic PA, the evolution of carboncarbon bond length of the anionic PA molecule under the external EF reverses. The LUMOHOMO gap of the anionic PA molecule decreases with the increase of the external EF. Under zero EF, both the HOMO and LUMO of all-trans PA molecule in neutral, cationic, and anionic states are delocalized on the whole molecular backbone. Under the influence of external EF, the HOMO and LUMO for the neutral PA slightly shift to low potential side and high potential side, respectively. The introduction of EF has almost no effect on the spatial distribution of the HOMO and LUMO of the cationic PA. With the application of external EF, both the HOMO and LUMO of anionic PA shift to high potential side. In particular, under certain EF the LUMO of anionic PA practically localized on the high potential end of the molecule.

\section{Acknowledgements}

We gratefully acknowledge the financial support of Guangxi Natural Science Foundation (No. 0991247), Guangxi Science Research and Technology Developing Foundation of China (No. 0842003-15) and Talent Development Foundation of Guilin University of Technology (No. 006206236).

\section{References}

[1] Friend R. H., Gymer R. W., Holmes A. B., Burroughes J. H., Marks R. N., Taliani C., Bradley D. D. C., Dos Santos D. A., Brédas J. L., Lögdlund M., Salaneck W. R.: Electroluminescence in conjugated polymers. Nature, 397, 121-128 (1999).

DOI: $10.1038 / 16393$

[2] Garcia A., Yang R., Jin Y., Walker B., Nguyen T.: Structure-function relationships of conjugated polyelectrolyte electron injection layers in polymer light emitting diodes. Applied Physics Letters, 91, 153502/1-153502/3 (2007).

DOI: $\underline{10.1063 / 1.2794422}$

[3] Horowitz G.: Organic field-effect transistors. Advanced Materials, 5, 365-377 (1998).

DOI: $10.1002 /(\mathrm{SICI}) 1521-4095(199803) 10: 5<365::$ AID-ADMA365>3.0.CO;2-U

[4] Sun Y., Liu Y., Zhu D.: Advances in organic fieldeffect transistors. Journal of Materials Chemistry, 15, 53-65 (2005). DOI: $\underline{10.1039 / \mathrm{b} 411245 \mathrm{~h}}$

[5] Akkerman H. B., Blom P. W. M., Leeuw D. M., Boer B.: Towards molecular electronics with large-area molecular junctions. Nature, 441, 69-72 (2006). DOI: $\underline{10.1038 / \text { nature04699 }}$

[6] Li Y., Yao J., Liu C., Yang C.: Theoretical investigation on electron transport properties of a single molecular diode. Journal of Molecular Structure: THEOCHEM, 867, 59-63 (2008). DOI: $\underline{10.1016 / \mathrm{j} . \text { theochem.2008.07.026 }}$ 
[7] Shirakawa H.: The discovery of polyacetylene film: The dawning of an era of conducting polymers. Synthesis Metals, 125, 3-10 (2001).

DOI: $\underline{10.1016 / \mathrm{S} 0379-6779(01) 00507-0}$

[8] Roth S., Filzmoser M.: Conducting polymers-thirteen years of Polyacetylene Doping. Advanced Materials, 21, 356-360 (2004). DOI: $10.1002 / \mathrm{adma} 19900020804$

[9] Lam J. W. Y., Luo J., Dong Y., Cheuk K. K. L., Tang B. Z.: Functional polyacetylenes: Synthesis, thermal stability, liquid crystallinity, and light emission of polypropiolates. Macromolecules, 35, 8288-8299 (2002).

DOI: $10.1021 / \mathrm{ma} 021011 \mathrm{a}$

[10] Pino R., Scuseria G. E.: Importance of chain-chain interactions on the band gap of trans-polyacetylene as predicted by second-order perturbation theory. Journal of Chemical Physics, 121, 8113-8119 (2004). DOI: $10.1063 / 1.1798991$

[11] Ciofini I., Adamo C., Chermette H.: Effect of selfinteraction error in the evaluation of the bond length alternation in trans-polyacetylene using density-functional theory. Journal of Chemical Physics, 123, 121102/1-121102/4 (2005). DOI: $\underline{10.1063 / 1.2047447}$

[12] Champagne B., Spassova M.: Structural properties of doped polyacetylene chains: A comparative theoretical investigation using Hartree-Fock, Moller-Plesset second-order perturbation theory, and density functional theory approaches. Physical Chemistry Chemical Physics, 6, 3167-3174 (2004).

DOI: $10.1039 / \mathrm{b} 402635 \mathrm{~g}$

[13] Brédas J. L.: Molecular geometry and nonlinear optics. Science, 263, 487-488 (1994). DOI: $10.1126 /$ science.263.5146.487

[14] Kertész M., Choi C. H., Yang S.: Conjugated polymers and aromaticity. Chemical Reviews, 105, 3448-3481 (2005). DOI: $10.1021 / \mathrm{cr} 990357 \mathrm{p}$

[15] Bundgaard E., Krebs F. C.: Low-band-gap conjugated polymers based on thiophene, benzothiadiazole, and benzobis(thiadiazole). Macromolecules, 39, 28232831 (2006). DOI: $10.1021 / \mathrm{ma} 052683 \mathrm{e}$

[16] Tang R., Tan Z., Li Y., Xi F.: Synthesis of new conjugated polyfluorene derivatives bearing triphenylamine moiety through a vinylene bridge and their stable blue electroluminescence. Chemistry of Materials, 18, 1053-1061 (2006).

DOI: $\underline{10.1021 / \mathrm{cm} 0522735}$

[17] Hou J., Huo L., He C., Yang C., Li Y.: Synthesis and absorption spectra of poly(3-(phenylenevinyl)thiophene)s with conjugated side chains. Macromolecules, 39, 594-603 (2006).

DOI: $\underline{10.1021 / \mathrm{ma} 051883 \mathrm{n}}$
[18] Sakaguchi H., Matsumura H., Gong H., Abouelwafa A. M.: Direct visualization of the formation of singlemolecule conjugated copolymers. Science, 310, 1002 1006 (2005).

DOI: $10.1126 /$ science. 1117990

[19] Chen F., Hihath J., Huang Z., Li X., Tao N. J.: Measurement of single-molecule conductance. The Annual Review of Physical Chemistry, 58, 535-564 (2007).

DOI: 10.1146/annurev.physchem.58.032806.104523

[20] Stan M. R., Franzon P. D., Goldstein S. C., Lach J. C., Ziegler M. M.: Molecular electronics: From devices and interconnect to circuits and architecture. Proceedings of the IEEE, 91, 1940-1957 (2003).

DOI: $10.1109 /$ JPROC.2003.818327

[21] Torrent-Sucarrat M., Sola M., Duran M., Luis J. M.: Basis set and electron correlation effects on ab initio electronic and vibrational nonlinear optical properties of conjugated organic molecules. Journal of Chemical Physics, 118, 711-718 (2003).

DOI: $\underline{10.1063 / 1.1521725}$

[22] Jacquemin D., Perpete E. A., Ciofini I., Adamo C.: Assessment of recently developed density functional approaches for the evaluation of the bond length alternation in polyacetylene. Chemical Physics Letters, 405, 376-381 (2005).

DOI: 10.1016/j.cplett.2005.02.037

[23] Li Y. W., Zhang Y., Yin G. P., Zhao J. W.: Theoretical investigations on molecular conducting wire under electric field. Chemical Journal of Chinese Universities, 27, 292-296 (2006).

[24] Frisch M. J., Trucks G. W., Schlegel H. B., Scuseria G. E., Robb M. A., Cheeseman J. R., Montgomery J. A., Vreven Jr., T., Kudin K. N., Burant J. C., Millam J. M., Iyengar S. S., Tomasi J., Barone V., Mennucci B., Cossi M., Scalmani G., Rega N., Petersson G. A., Nakatsuji H., Hada M., Ehara M., Toyota K., Fukuda R., Hasegawa J., Ishida M., Nakajima T., Honda Y., Kitao O., Nakai H., Klene M., Li X., Knox J. E., Hratchian H. P., Cross J. B., Adamo C., Jaramillo J., Gomperts R., Stratmann R. E., Yazyev O., Austin A. J., Cammi R., Pomelli C., Ochterski J. W., Ayala P. Y., Morokuma K., Voth G. A., Salvador P., Dannenberg J. J., Zakrzewski V. G., Dapprich S., Daniels A. D., Strain M. C., Farkas O., Malick D. K., Rabuck A. D., Raghavachari K., Foresman J. B., Ortiz J. V., Cui Q., Baboul A. G., Clifford S., Cioslowski J., Stefanov B. B., Liu G., Liashenko A., Piskorz P., Komaromi I., Martin R. L., Fox D. J., Keith T., Al-Laham M. A., Peng C. Y., Nanayakkara A., Challacombe M., Gill P. M. W., Johnson B., Chen W., Wong M. W., Gonzalez C., Pople J. A.: Gaussian 03, Revision C.02, Gaussian, Inc., Wallingford, USA (2004).

[25] Schön J. H., Meng H., Bao Z.: Field-effect modulation of the conductance of single molecules. Science, 294, 2138-2140 (2001).

DOI: $\underline{10.1126 / \text { science. } 1066171}$ 
[26] Champagne B., Perpete E. A.: Bond length alternation effects on the static electronic polarizability and second hyperpolarizability of polyacetylene chains. International Journal of Quantum Chemistry, 75, 441-447 (1999).

DOI: $10.1002 /($ SICI $) 1097-461 X(1999) 75: 4 / 5<441::$ AID-QUA10>3.0.CO;2-B

[27] Marder S. R., Gorman C. B., Tiemann B. G., Perry J. W., Bourhill G., Mansour K.: Relation between bondlength alternation and second electronic hyperpolarizability of conjugated organic molecules. Science, 261, 186-189 (1993).

DOI: $10.1126 /$ science. 261.5118 .186

[28] Brisset H., Blanchard P., Illien B., Riou A., Roncali J.: Bandgap control through reduction of bond length alternation in bridged poly(diethienylethylene)s. Chemical Communications, 569-570 (1997). DOI: $10.1039 / \mathrm{a} 607890 \mathrm{~g}$

[29] Champagne B., Deumens E., Öhrn Y.: Vibrations and soliton dynamics of positively charged polyacetylene chains. The Journal of Chemical Physics, 107, 54335444 (1997).

DOI: $10.1063 / 1.474249$
[30] Zhang Y., Ye Y., Li Y., Yin X., Liu H., Zhao J.: Ab initio investigations of quaterthiophene molecular wire under the interaction of external electric field. Journal of Molecular Structure: THEOCHEM, 802, 53-58 (2007).

DOI: 10.1016/j.theochem.2006.09.012

[31] Li Y., Zhao J., Yin G.: Theoretical investigations of oligo(phenylene ethylene) molecular wire: Effects from substituents and external electric field. Computational Materials Science, 39, 775-781 (2007). DOI: $\underline{10.1016 / \text { j.commatsci.2006.09.010 }}$

[32] Aihara J.: Correlation found between the HOMOLUMO energy separation and the chemical reactivity at the most reactive site for isolated-pentagon isomers of fullerenes. Physical Chemistry Chemical Physics, 2, 3121-3125 (2000). DOI: $10.1039 / \mathrm{b} 002601 \mathrm{~h}$

[33] Singh T. B., Sariciftci N. S.: Progress in plastic electronics devices. Annual Review of Materials Research, 36, 199-230 (2006). DOI: 10.1146/annurev.matsci.36.022805.094757

[34] Li Y., Zhao J., Yin X., Liu H., Yin G.: Conformational analysis of diphenylacetylene under the influence of an external electric field. Physical Chemistry Chemical Physics, 9, 1186-1193 (2007).

DOI: $\underline{10.1039 / \mathrm{b} 615618 \mathrm{e}}$ 